
.

\title{
A High Serum Level of Taurocholic Acid is Correlated with the Severity and
}

\section{Resolution of Drug-induced Liver Injury}

\section{Short title: Taurocholic acid and the severity of DILI}

Qiuju Tian ${ }^{1 \#}$, Ruiyuan Yang ${ }^{1 \#}$, Yan Wang ${ }^{1}$, Jimin $\mathrm{Liu}^{2}$, Aileen Wee ${ }^{3}$, Romil Saxena ${ }^{4}$, Lan Wang ${ }^{1}$, Min $\mathrm{Li}^{5}$, Liwei Liu ${ }^{1}$, Shan Shan ${ }^{1}$, Yuanyuan Kong ${ }^{5}$, Hong Ma ${ }^{1}$, Xiaojuan $\mathrm{Ou}^{1}$, Hong You ${ }^{1}$, Xinyan Zhao ${ }^{1}$, Jidong $\mathrm{Jia}^{1}{ }^{*}$

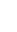

${ }^{1}$ Liver Research Center, Beijing Friendship Hospital, Capital Medical University, National Clinical Research Center for Digestive Diseases, Beijing, China

${ }^{2}$ Department of Pathology and Molecular Medicine, Faculty of Health Sciences, McMaster University, Hamilton, Ontario, Canada

${ }^{3}$ Department of Pathology, Yong Loo Lin School of Medicine, National University of Singapore, National University Hospital, Singapore, Singapore

${ }^{4}$ Department of Pathology, Indiana University School of Medicine, Indianapolis, Indiana, USA

${ }^{5}$ Clinical Epidemiology and Evidence-Based Medicine Unit, National Clinical Research Center for Digestive Diseases, Beijing Friendship Hospital, Capital Medical University, Beijing, China

Grant support: This work was funded by grants from the National Natural Science Foundation of China (No. 81670545), the Digestive Medical Coordinated

This is the author's manuscript of the article published in final edited form as: 
Development Center of Beijing Hospitals Authority (No. XXZ0301), the National

Science and Technology Major Special Project for New Drug Development (No.

2018ZX09201016) and the Key Technical and Executive Measures to Improve Early

Phase Clinical Trials on Innovative Drugs for Liver Diseases (No.

Z191100007619037).

\section{Abbreviations:}

ABCB11, ATP Binding Cassette Subfamily B Member 11; ALF, acute liver failure;

BA, bile acid; BSEP, bile salt export pump; CHB, chronic hepatitis B; DILI, drug-induced liver injury; TCA, taurocholic acid; ULN, upper limits of normal.

\section{${ }^{\#}$ Qiuju Tian and Ruiyuan Yang contributed equally to this work.}

\section{*Correspondence to:}

Xinyan Zhao, MD, Ph.D. and Jidong Jia, MD, Ph.D.

Liver Research Center, Beijing Friendship Hospital, Capital Medical University, 95

Yong-An Road, Xicheng District, Beijing 100050, China.

Tel: +8610 63138435; Fax: +861063169246

E-mail: zhao_xinyan@ccmu.edu.cn (XZ); jia_jd@ccmu.edu.cn $(J J)$

\section{Author's contributions:}

Study concept and design: JJ, XZ

Acquisition of data: QT, RY, XZ, JL, YW, SS, LW, LL, HM, HY, XO 
45 Statistical Analysis and interpretation of data: QT, RY, XZ, ML, YK

46 Drafting of the manuscript: QT

47 Critical revision of the manuscript for important intellectual content: JJ, XZ, JL, AW, 48 RS, YW

49 Obtained funding and study supervision: JJ, XZ

50

51 Disclosures: All authors declare that there are no conflicts of interests in this work.

52

53 Acknowledgements: We are deeply grateful to Wei Chen, Donghu Zhou, and Siyu Jia

54 from Experimental and Translational Research Center, Beijing Friendship Hospital,

55 Capital Medical University, Beijing, China, for their help in the experiments and 56 interpretation of the results, and to Dr. Ipsita Panda from India for her generous help 57 in critical revision and language polishing. 


\section{Abstract}

Background \& Aims: Alterations in the serum levels of bile acids are associated with drug-induced liver injury (DILI). We investigated the association between serum levels of bile acids and the severity and outcome of DILI, along with the potential role of variants in the ATP binding cassette subfamily B member $11(A B C B 11)$ gene and expression of its product, ABCB11 (also called BSEP).

Methods: We performed this prospective study of 95 patients (median age, 53 years; 73.7\% female) with DILI from August 2018 through August 2019. Patients were matched for age, gender, and body mass index with healthy individuals $(n=100$; healthy controls) and patients with chronic hepatitis B ( $\mathrm{n}=105$; CHB controls). We collected demographic and biochemical data at baseline and 1 week, 1 month, 3 months, and 6 months after DILI onset and at the time of biochemical recovery, liver failure or liver transplantation. Serum levels of bile acids were measured using high-performance liquid-chromatography tandem mass-spectrometry. All 27 exons of $A B C B 11$ were sequenced and expression of BSEP were analyzed by immunohistochemistry in liver biopsy specimens.

Results: Levels of 30 of the 37 bile acids analyzed differed significantly between patients with DILI and healthy controls. Changes in levels of taurocholic acid (TCA), glycocholic acid, taurochenodeoxycholate, and glycochenodeoxycholate associated with the increased levels of bilirubin and greater severity of DILI, and were also associated with CHB. Cox regression analysis showed that only change in the levels of TCA independently associated with biochemical resolution of DILI. Combination of TCA level ( $\geq 1955.41 \mathrm{nmol} / \mathrm{L})$, patient age, and DILI severity was associated with abnormal blood biochemistry at 6 months after DILI onset (area under the curve, 0.81; 95\% confidence interval, $0.71-0.88$; sensitivity, 0.69 ; specificity, 0.81). ABCB11 

$\overline{\text { missense variants were not associated with differences in the serum bile acid profiles, }}$ DILI severity, or clinical resolution. However, lower levels of BSEP in bile canaliculi

86 in liver biopsies were associated with altered serum levels of bile acids.

87 Conclusions: In this prospective study performed in Chinese patients, we found that 88 the serum levels of TCA were associated with the severity and clinical resolution of 89 DILI. Reduced protein expression of BSEP in liver tissue, rather than variants of the $90 A B C B 11$ gene were associated with altered serum levels of bile acids.

91 Key words: toxicity; herbal supplement; bile salt export pump; hepatitis B virus 92 


\section{Introduction}

Drug-induced liver injury (DILI) is one of the most common causes of clinically significant liver injury, with an annual incidence of $14-24$ per 100,000 person-years worldwide $^{[1-3]}$. DILI can have different phenotypes with diverse clinical features and variable outcomes. Cholestatic DILI is more prone to chronicity ${ }^{[4-6]}$, whereas hepatocellular DILI has greater severity ${ }^{[7]}$ and higher mortality once jaundice develops ${ }^{[8-11]}$. Although $87 \%$ of DILI patients achieve biochemical resolution within 6 months after withdrawal of the offending $\operatorname{drug}(\mathrm{s})^{[3]}, 8 \%$ still have biochemical abnormalities within 12 months $^{[12]}$, leading to chronicity or even cirrhosis. Histological characteristics could aid in the prediction of clinical outcomes ${ }^{[4-6,13,14]}$. However, other noninvasive serum biomarkers are urgently needed in routine clinical practice.

Recent evidence has shown that bile acid (BA) dysregulation plays a pivotal role in DILI, as bile excretion is the major route to eliminate bilirubin and some potentially harmful exogenous lipophilic substances ${ }^{[15,16]}$. Animal studies have highlighted the diagnostic, prognostic and therapeutic importance of BAs in DILI ${ }^{[17-22]}$. In a human study, serum glycodeoxycholic acid level showed prognostic value in acetaminophen-induced acute liver failure $(\mathrm{ALF})^{[23]}$. More recently, Ma et al. found that the serum BA levels could serve as potential biomarkers for the diagnosis and the distinction of mild from severe DILI ${ }^{[24]}$.

However, the association of altered BA profiles with the severity and prognosis of DILI warrants further verification. In addition, the potential mechanism of this 
probable association remains unknown, although previous studies have illustrated the

117 essential role of the bile salt export pump (BSEP), encoded by the ATP binding

118 cassette subfamily B member $11(A B C B 11)$ gene, in DILI ${ }^{[25,26]}$.

119 In this prospective study, we investigated the differences in BA profiles in DILI

120 patients, healthy controls, and chronic hepatitis B (CHB) patients. Then, we explored

121 the association of altered BA profiles with clinical phenotypes and the severity and resolution of DILI. Finally, we explored the potential roles of $A B C B 11$ gene variants

123 and the liver expression of ABCB11 (BSEP) in the pathogenesis of altered serum BA 124 profiles. 
126

127

128

129

130

131

132

133

134

135

136

137

138

139

140

141

142

143

144

145

146

147

Materials and Methods

\section{Study population}

In this single-center cohort study, we prospectively recruited patients with a clinical diagnosis of DILI at Beijing Friendship Hospital, Capital Medical University, from August 2018 to August 2019.

The inclusion criteria were as follows: (1) Chinese Han nationality aged 18 to 78 years; (2) outpatients or inpatients with a diagnosis of DILI; and (3) available blood samples from the acute phase (within 10 days of obtaining the peak value of transaminase or bilirubin).

The exclusion criteria were as follows: (1) other underlying liver disease or systemic diseases affecting the liver; (2) treatment with ursodeoxycholic acid, steroids, antibiotics or probiotics two weeks prior to the collection of blood samples; (3) a steroid-dependent state; (4) cirrhosis; and (5) loss to follow-up before the study endpoints.

The diagnosis of DILI was re-ascertained ${ }^{[9]}$ by three hepatologists (QT, RY, and LW) using Roussel Uclaf Causality Assessment Method (RUCAM) score $\geq 3$ ("possible", "probable" or "highly probable" DILI). Based on the R value [ratio of alanine aminotransferase and alkaline phosphatase with their upper limits of normal (ULN)] at disease onset, DILI patients were categorized as having hepatocellular $(\mathrm{R} \geq 5)$, cholestatic $(\mathrm{R} \leq 2)$, or mixed $(2<\mathrm{R}<5)$ biochemical phenotypes ${ }^{[8]}$. The disease severity was evaluated according to previously reported criteria ${ }^{[9]}$.

Follow-up information was collected at 1 week, 1 month, 3 months, and 6 months 
after onset until biochemical recovery ${ }^{[6]}$. The clinical outcomes in this study included biochemical recovery, liver failure or liver transplantation.

Age-, gender- and body mass index-matched healthy individuals and a CHB cohort at the same hospital were enrolled as controls (Supplementary Material 1).

This study was approved by the Institutional Ethical Review Board of the Beijing Friendship Hospital, Capital Medical University [2018-P2-116-02]. All participants gave informed consent.

\section{Measurements of serum BAs}

Fasting serum samples from DILI patients during the acute phase and samples obtained from healthy controls and CHB patients were collected and frozen at $-80^{\circ} \mathrm{C}$ for the subsequent BA measurement. Comprehensive quantitation of serum BAs was performed with high-performance liquid chromatography tandem mass spectrometry $^{[27]}$ at Metabo-Profile Inc. (Shanghai, China), which was blinded to any clinical information (Supplementary Material 2).

\section{Sanger sequencing of all 27 coding exons of the $A B C B 11$ gene}

Peripheral blood mononuclear cells from the enrolled DILI patients and healthy controls were collected. Forward and reverse Sanger sequencing of all 27 coding exons of the $A B C B 11$ gene and the associated boundary regions (adjacent sequences in introns) were conducted (Supplementary Material 3 \& Supplementary Table 1).

\section{Immunohistochemical evaluation of BSEP}

Percutaneous liver biopsies of DILI patients were fixed in formalin, embedded in paraffin, and sectioned at a thickness of $4 \mu \mathrm{m}$. BSEP expression was evaluated with 
170

171

172

173

174

175

176

177

178

179

180

181

182

183

184

185

186

immunohistochemistry with antibody obtained from Santa Cruz Biotechnology (Shanghai) Co., Ltd., China (Supplementary Material 4).

Semiquantitative evaluation of BSEP staining was conducted by two liver pathologists (XZ and JL) microscopically. Normal expression was defined as no loss or a mild loss $(\leq 1 / 3)$ of BSEP expression, and reduced expression was defined as an obvious loss (>1/3) of BSEP expression. Quantitative evaluation of BSEP expression with Image-Pro Plus 6.0 (Media Cybernetics, Inc.) was conducted by a hepatologist (QT) with pathology training who was blinded to the patient data.

\section{Statistical analysis}

Categorical variables were expressed as counts or percentages, and continuous variables were expressed as the means \pm standard deviations or medians and interquartile ranges. Comparisons of different groups were performed using the chi-square test, analysis of variance or the Kruskal-Wallis test; a Cox regression model was used to identify potential risk factors associated with clinical resolution, and Kappa statistics were used to assess the agreement. These analyses were conducted with SPSS 24.0 (SPSS Inc., Chicago, IL).

The bioinformatic analysis was conducted using R 3.6.1 (R Foundation for Statistical Computing Platform).

The difference in diagnostic performance was assessed using receiver operating characteristic (ROC) curves; the area under the ROC (AUROC) values were compared with the Delong method using MedCalc 12.2.1.0 (MedCalc, Mariakerke, Belgium). 
For all analyses, $P<0.05$ was considered statistically significant. 
193

194

195

196

197

198

199

200

201

202

203

204

205

206

207

208

209

210

211

212

213

214
Results

Clinical characteristics of DILI patients, healthy controls and CHB patients

This study included 95 DILI patients, 100 healthy controls (Fig. 1) and 105 noncirrhotic $\mathrm{CHB}$ patients. Age, gender, and BMI were comparable among three groups. The demographic, biochemical and clinical characteristics are summarized in Table 1 and Supplementary Table 2-3.

The median age of DILI patients was 53 years, and the majority (73.7\%) were females. Based on the $\mathrm{R}$ value, 63, 17 and 15 cases were defined as having a hepatocellular, cholestatic and mixed phenotype, respectively. According to the severity evaluation, all patients were categorized as 38 mild (Grade 1), 13 moderate (Grade 2), 40 severe (Grade 3), 3 liver failure (Grade 4) and 1 fatal (Grade 5).

The median RUCAM score was 6 . The majority $(74.7 \%)$ of DILI cases were attributed to the use of herbs (Supplementary Material 5 and Supplementary Table 4). The median biochemical recovery time was 88 days; 79 cases resolved at 6 months, and 86 cases resolved at 12 months.

The BA profiles in DILI patients were significantly different from those in healthy controls

As illustrated by the principal component analysis (PCA) (Supplementary Fig. 1A), distinct clustering with minimal overlap was observed between DILI patients and healthy controls. Of the 37 measured BAs, the levels of 30 BAs were significantly different between DILI patients and healthy controls (Supplementary Table 5). The top six most significantly changed BAs with the strongest predictive ability (AUROC > 
0.96) for distinguishing DILI patients from healthy controls are depicted in

Supplementary Fig. $1 B$ \& C.

BA profiles were comparable among DILI patients with different biochemical

\section{phenotypes and with different insulting agents}

DILI patients with hepatocellular, cholestatic and mixed phenotypes were not be distinguished among the three phenotypes based on the PCA plot (Fig. 2A).

Further analysis of the level of each BA showed that they were generally comparable across the three phenotypes.

Details of insulting agents are listed in Supplementary Table 4. We divided the

DILI patients into three groups according to the types of insulting agents: herbs, conventional medicine and a combination of herbs and conventional medicine. As the PCA plot showed, the serum BA profiles in these groups largely overlapped groups.

BA levels increased with the serum bilirubin level and could be used to

\section{distinguish the severity of DILI}

The levels of five BAs, namely, taurocholic acid (TCA), glycocholic acid (GCA),

234 glycoursodeoxycholic acid (GUDCA), showed an increasing trend along with the 235 serum bilirubin levels (total bilirubin <5 ULN, 5-10 ULN, 10-20 ULN and >20 ULN) 
Further analysis of the BA profiles in the mild $(\mathrm{N}=38)$, moderate $(\mathrm{N}=13)$, and severe/liver failure/fatal $(\mathrm{N}=44)$ groups of DILI patients revealed that a subgroup of BAs could distinguish different groups, as shown in the Venn diagram (Fig. 2C), and the details regarding the BAs are listed in Supplementary Table 6. Specifically, TCA,

241 GCA, TCDCA and GCDCA could credibly determine the degree of DILI severity, as 242 they proportionately rose with an increase in severity (Fig. 2D).

Notably, four patients with dismal prognoses had strikingly high TCA levels: $10152.46 \mathrm{nmol} / \mathrm{L}$ (ALF; received artificial liver support), $13742.13 \mathrm{nmol} / \mathrm{L}$ (ALF; recovered 66 days after drug withdrawal), $28537.60 \mathrm{nmol} / \mathrm{L}$ (ALF; received artificial liver support) and $26136.74 \mathrm{nmol} / \mathrm{L}$ (received liver transplantation), further 247 corroborating the prognostic implications of serum TCA in DILI patients, as the median value of TCA in the whole DILI cohort was $1955.41 \mathrm{nmol} / \mathrm{L}$.

Serum TCA was an independent factor that predicted the timing of disease resolution and could improve the prediction of the 6-month outcome in DILI patients

We assessed the role of clinical features (age, gender, BMI, and severity) and BA 253 profiles in predicting the clinical course (days to biochemical normalization) with 254 proportional Cox regression. TCA was found to be the only independent factor 255 associated with biochemical resolution (Table 2).

256 We then analyzed the predictive ability of age, severity, TCA, and their 257 combination for assessing the 6-month outcome (abnormal biochemistry). The 258 AUROC values for age, severity, TCA, and their combination were $0.58,0.65,0.69$ 
and 0.81, respectively (Fig. 2E; Table 3).

High serum TCA level alone could be a potential useful indicator for predicting 6-month abnormal biochemistry outcome with an optimal cut-off value of 1955.41 $\mathrm{nmol} / \mathrm{L}(\sim 2 \mu \mathrm{mol} / \mathrm{L})(\mathrm{AUROC}=0.69$, sensitivity=0.81, specificity=0.57). Furthermore, the combination of TCA, severity and age had a better predictive ability for the 6-month outcome, with an AUROC value of 0.81 and a sensitivity and specificity of 0.69 and 0.81, respectively (Table 3).

\section{Changes in serum BAs along with serum bilirubin were also observed in CHB} patients

The levels of several BAs, including TCA, were significantly different among healthy controls, CHB patients and DILI patients. They showed a stepwise significant increase in CHB and DILI patients compared with those in healthy controls (Supplementary Table 7).

Totally, the levels of seven BAs were increased along with serum bilirubin in CHB patients (Supplementary Table 8). Four out of these seven BAs, i.e., TCA, GCA, TCDCA, and GCDCA, were also increased along with serum bilirubin in DILI patients (Supplementary Fig. 3).

Although the TCA level was significantly lower in CHB patients than that in DILI patients, its increase along with serum bilirubin was similar to that observed in DILI patients. In addition, three CHB patients with acute on chronic liver failure had extremely high serum TCA levels of $15101.88 \mathrm{nmol} / \mathrm{L}, 11734.34 \mathrm{nmol} / \mathrm{L}$ and 7621.98 nmol/L, while the median value of TCA level in the CHB cohort was $80.14 \mathrm{nmol} / \mathrm{L}$. 
These results suggested that TCA was increased along with serum bilirubin and may be associated with liver failure without DILI specificity.

283

Genetic variants of $A B C B 11$ did not account for serum BA alterations or the severity or clinical resolution of DILI

The genetic variants in $A B C B 11$ gene are listed in Supplementary Table 9. The frequencies of all $A B C B 11$ missense variants in DILI patients were not significantly different from those in healthy Chinese controls. A total of 71 DILI patients (74.7\%) had missense variants of $A B C B 11$; however, they had comparable serum BA profiles, severity scores, and time to resolution with the remaining 24 DILI cases not having any $A B C B 11$ missense variant (Fig. 3A \& B).

Reduced liver expression of BSEP was associated with serum BA alterations and disease severity

Liver biopsies were performed in 34 of the 95 DILI patients. The biopsied patients had comparable baseline characteristics with the remaining patients who declined biopsy (Supplementary Table 10). Hence, the biopsied patients were considered representative of the entire DILI cohort. Based on semiquantitative assessment by the liver pathologists, BSEP expression was ranked as either normal or reduced (Fig. 3C \& D). The semiquantification of BSEP expression was validated by quantification analysis (indexed as the mean integrated optical density using Image-Pro Plus), which resulted in good interobserver agreement (kappa value 0.90).

The 11 DILI cases with reduced BSEP expression had significantly higher BA levels (including TCA, GCA and GCDCA) compared with the remaining 23 cases 
303 with normal BSEP expression (Fig. 3E). In addition, severe DILI was observed in 64\%

304 versus $26 \%$ of DILI cases with reduced and normal BSEP expression, respectively 305 (one-sided $P=0.042$ ) (Fig. 3F). 
306

\section{Discussion}

In this study, we found that the levels of certain conjugated BAs increased in a stepwise manner along with DILI severity. Furthermore, the level of serum TCA was independently associated with the clinical resolution of DILI. The alteration of serum BAs was associated with reduced liver expression of BSEP but not with genetic variants of $A B C B 11$, encoding BSEP. Taken together, these results suggest that a high serum TCA level is positively associated with disease severity and can potentially predict the clinical resolution of DILI patients.

In our study, TCA, GCA, TCDCA and GCDCA were found to increase along with disease severity. This is in line with a previous study that showed similar disruptions of BA levels, serving as biomarkers for the differentiation of severe and mild DILI ${ }^{[24]}$. Similarly, Woolbright et al. found that serum glycodeoxycholic acid could predict the survival of acetaminophen-induced ALF patients ${ }^{[23]}$. All these evidences shed light on the role of BAs in identifying high-risk DILI. Our study suggested that routinely serum test for these BAs would aid in the identification of DILI patients with greater severity or at higher risk of liver failure so that additional supportive/preventive measures could be initiated.

Moreover, our study revealed that the TCA level, as tested during the acute phase, was an independent risk factor for biochemical resolution in DILI. The TCA level alone had a similar predictive performance for the 6-month abnormal biochemistry outcome as the current constellation of severity assessment. Since the DILI severity discrimination is based on a group of parameters, including: symptoms, serum 
bilirubin, blood clotting tests, hospitalization and organ dysfunction [9, 10, 28-30], measurement of the serum TCA level may serve as a new biomarker for DILI stratification and outcome prediction. In fact, when TCA measurement was added to the current outcome assessment, the predictive performance for DILI resolution improved from 0.75 for severity-age to $0.81^{[31]}$ for TCA-severity-age, although the difference was not statistically significant. It is worth noting that the increase in serum TCA, GCA, TCDCA and GCDCA along with serum bilirubin in both DILI and CHB patients might suggest that the alteration of these BAs was not disease-specific.

In addition, we found that missense variants of $A B C B 11$ were not associated with serum BA alterations, disease severity or days to resolution in DILI. This finding was also consistent with those of large-cohort genome-wide association studies, which did not find genetic susceptibility of $A B C B 11$ in multiple-drug-induced liver injury ${ }^{[32,33]}$. This may serve as a strong evidence that DILI is indeed an acquired instead of inborn 341 disease ${ }^{[34]}$. Nevertheless, we did show that the alteration of BA profiles was partially 342 due to the reduced expression of BSEP, a protein encoded by the $A B C B 11$ gene and 343 the molecule responsible for pumping bile salts from the hepatocellular cytoplasm 344 into the bile canaliculi ${ }^{[16]}$. Our findings suggest that reduced protein expression of 345 BSEP in DILI patients may occur during transcriptional and/or translational processes 346 and/or due to the interactions between the host and the drug ${ }^{[35,36]}$, which warrants 347 further in-depth investigation.

348 The strength of this study is that it offers clinical evidence that routine 349 measurement of certain serum BAs may improve the identification of high-risk DILI 
patients and the prediction of clinical outcomes. Furthermore, we revealed that the underlying mechanism of the altered $\mathrm{BA}$ profiles was not due to $A B C B 11$ genetic variants but instead to reduced BSEP expression at the protein level.

However, the conclusion from this single-center prospective study of Chinese Han individuals with a moderate sample size needs further validation in multicenter studies. Furthermore, only $36 \%$ of the entire DILI cohort had a liver biopsy available for BSEP analysis, which may introduce possible selection bias. However, there was no significant difference in the demographic and major clinical profiles between those with and without liver biopsies; thus, we hope the BSEP immunohistochemical staining results to be interpreted as representative of the entire DILI cohort.

In conclusion, the serum levels of the conjugated BAs TCA, GCA, TCDCA and GCDCA showed a strong association with the severity of DILI. Specifically, TCA is an important biomarker that is associated with clinical resolution and can improve the prediction of the 6-month biochemical resolution outcome. The alteration of BA profiles is not due to $A B C B 11$ genetic variants but is due to reduced BSEP protein expression. The present findings provide further evidence that determination of the BA profile may help to optimize the management of DILI in clinical practice. 


\section{Figure Legends:}

\section{Fig. 1 Enrollment of DILI patients and healthy control subjects. ${ }^{1}$}

\section{Fig. 2 The exploration of BAs in association with severity and days to resolution.}

Overall, the levels of serum BAs in DILI patients with different biochemical phenotypes could not be distinguished as separate clusters via principal component analysis (A); TCA, GCA, TCDCA, GCDCA and GUDCA levels showed gradually increasing trends along with serum bilirubin levels (B); a subgroup of BAs could distinguish different severities of DILI (C); TCA, GCA, TCDCA and GCDCA rose in proportion with the increases of severity (D); the diagnostic performance of different indexes for predicting the 6-month abnormal biochemistry (E). ${ }^{2}$

Fig. 3 Role of $A B C B 11 / B S E P$ in $\mathrm{BA}$ profiles, severity, and days to resolution in DILI patients. Overall BA levels were comparable in DILI patients with or without $A B C B 11$ missense variants (A); DILI patients with or without $A B C B 11$ missense variants had comparable severities and days to resolution $(\mathbf{B})$; examples of normal $(\mathbf{C})$ and reduced (D) BSEP expression (immunohistochemical staining, 200x); DILI patients with reduced BSEP expression showed greater alteration of BA profiles compared with those with normal BSEP expression(E) and a higher risk of having a severe case $(\mathbf{F})$; however, they had a similar number of days to resolution. No significant difference in BSEP expression was found between patients with and

\footnotetext{
${ }^{1}$ HBV, hepatitis B virus; NAFLD, nonalcoholic fatty liver disease; PBC, primary biliary cholangitis, SSC, secondary sclerosing cholangitis.

2 GCA, glycocholic acid; GCDCA, glycochenodeoxycholate; GUDCA, glycoursodeoxycholic acid; PC, principal component; TBil, total bilirubin; TCA, taurocholic acid; TCDCA, taurochenodeoxycholate; ULN, upper limit of normal.
} 
without $A B C B 11$ missense variants. ${ }^{3}$

388

${ }^{3}$ GCA, glycocholic acid; GCDCA, glycochenodeoxycholate; GHCA, glycohyocholate; Missense (-), DILI patients without any $A B C B 11$ missense variant(s); Missense (+), DILI patients with ABCB11 missense variant(s); Normal BSEP, DILI patients with normal BSEP expression; Reduced BSEP, DILI patients with reduced BSEP expression; TCA, taurocholic acid. 


\section{References:}

390 1. Sgro C, Clinard F, Ouazir K, et al. Incidence of drug-induced hepatic injuries: a 391 French population-based study. Hepatology 2002;36:451-455.

2. Bjornsson ES, Bergmann OM, Bjornsson HK, et al. Incidence, presentation, and 393 outcomes in patients with drug-induced liver injury in the general population of 394 Iceland. Gastroenterology 2013;144:1419-1425, 1421-1425, e19-e20.

3. Shen T, Liu Y, Shang J, et al. Incidence and Etiology of Drug-Induced Liver Injury 396 in Mainland China. Gastroenterology 2019;156:2230-2241.

4. Bonkovsky HL, Kleiner DE, Gu J, et al. Clinical presentations and outcomes of 398 399 bile duct loss caused by drugs and herbal and dietary supplements. Hepatology 2017;65:1267-1277.

400 5. Bjornsson ES, Jonasson JG. Idiosyncratic drug-induced liver injury associated 401 with bile duct loss and vanishing bile duct syndrome: Rare but has severe consequences. 402 Hepatology 2017;65:1091-1093.

403 6. Tian QJ, Zhao XY, Wang Y, et al. Histologic pattern is better correlated with 404 clinical outcomes than biochemical classification in patients with drug-induced liver 405 injury. Mod Pathol 2019;32:1795-1805.

406 7. Andrade RJ, Lucena MI, Kaplowitz N, et al. Outcome of acute idiosyncratic 407 drug-induced liver injury: Long-term follow-up in a hepatotoxicity registry. 408 Hepatology 2006;44:1581-1588.

409 8. Hoofnagle JH, Bjornsson ES. Drug-Induced Liver Injury - Types and Phenotypes. $410 \quad$ N Engl J Med 2019;381:264-273. 
9. Yu YC, Mao YM, Chen CW, et al. CSH guidelines for the diagnosis and treatment

412 of drug-induced liver injury. Hepatol Int 2017;11:221-241.

413 10. Chalasani NP, Hayashi PH, Bonkovsky HL, et al. ACG Clinical Guideline: the 414 diagnosis and management of idiosyncratic drug-induced liver injury. Am J 415 Gastroenterol 2014;109:950-966, 967.

416 11. Kullak-Ublick GA, Andrade RJ, Merz M, et al. Drug-induced liver injury: recent 417 advances in diagnosis and risk assessment. Gut 2017;66:1154-1164.

418 12. Medina-Caliz I, Robles-Diaz M, Garcia-Munoz B, et al. Definition and risk factors 419 for chronicity following acute idiosyncratic drug-induced liver injury. J Hepatol $420 \quad 2016 ; 65: 532-542$.

421 13. Wang $\mathrm{T}$, Zhao $\mathrm{X}$, Shao $\mathrm{C}$, et al. A proposed pathologic sub-classification of 422 drug-induced liver injury. Hepatol Int 2019;13:339-351.

423 14. Kleiner DE, Chalasani NP, Lee WM, et al. Hepatic histological findings in 424 suspected drug-induced liver injury: systematic evaluation and clinical associations. 425 Hepatology 2014;59:661-670.

426 15. Mosedale M, Watkins PB. Drug-induced liver injury: Advances in mechanistic 427 understanding that will inform risk management. Clin Pharmacol Ther $428 \quad 2017 ; 101: 469-480$.

429 16. Boyer JL. Bile formation and secretion. Compr Physiol 2013;3:1035-1078.

430 17. Yang L, Xiong A, He Y, et al. Bile acids metabonomic study on the CCl4- and 431 alpha-naphthylisothiocyanate-induced animal models: quantitative analysis of 22 bile 432 acids by ultraperformance liquid chromatography-mass spectrometry. Chem Res 
433

434 435 436 438 439

Toxicol 2008;21:2280-2288.

18. Cepa S, Potter D, Wong L, et al. Individual serum bile acid profiling in rats aids in human risk assessment of drug-induced liver injury due to BSEP inhibition. Toxicol Appl Pharmacol 2018;338:204-213.

19. Slopianka M, Herrmann A, Pavkovic M, et al. Quantitative targeted bile acid profiling as new markers for DILI in a model of methapyrilene-induced liver injury in rats. Toxicology 2017;386:1-10.

20. Luo L, Schomaker S, Houle C, et al. Evaluation of serum bile acid profiles as biomarkers of liver injury in rodents. Toxicol Sci 2014;137:12-25.

21. Paridaens A, Raevens S, Colle I, et al. Combination of tauroursodeoxycholic acid and $\mathrm{N}$-acetylcysteine exceeds standard treatment for acetaminophen intoxication. Liver Int 2017;37:748-756.

22. Fickert $\mathrm{P}$, Wagner M. Biliary bile acids in hepatobiliary injury - What is the link? $\mathrm{J}$ Hepatol 2017;67:619-631.

23. Woolbright BL, McGill MR, Staggs VS, et al. Glycodeoxycholic acid levels as prognostic biomarker in acetaminophen-induced acute liver failure patients. Toxicol Sci 2014;142:436-444.

24. Ma Z, Wang X, Yin P, et al. Serum metabolome and targeted bile acid profiling reveals potential novel biomarkers for drug-induced liver injury. Medicine (Baltimore) 2019;98:e16717.

25. Kenna JG, Taskar KS, Battista C, et al. Can Bile Salt Export Pump Inhibition Testing in Drug Discovery and Development Reduce Liver Injury Risk? An 
455

456

457 458

459

460

461

462

463

464

465

466

467 468

469

470

471 Dis 2020;20:304.

472

473

474

475

476 2018;104:916-932. 2009;32:55-68. 2019;70:1222-1261. Dis 2020,20:304. 2012;22:784-795.

International Transporter Consortium Perspective. Clin Pharmacol Ther

26. Aleo MD, Luo Y, Swiss R, et al. Human drug-induced liver injury severity is highly associated with dual inhibition of liver mitochondrial function and bile salt export pump. Hepatology 2014;60:1015-1022.

27. Xie G, Wang Y, Wang X, et al. Profiling of serum bile acids in a healthy Chinese population using UPLC-MS/MS. J Proteome Res 2015;14:850-859.

28. Fontana RJ, Watkins PB, Bonkovsky HL, et al. Drug-Induced Liver Injury Network (DILIN) prospective study: rationale, design and conduct. Drug Saf

29. Aithal GP, Watkins PB, Andrade RJ, et al. Case definition and phenotype standardization in drug-induced liver injury. Clin Pharmacol Ther 2011;89:806-815.

30. EASL Clinical Practice Guidelines: Drug-induced liver injury. J Hepatol

31. Dittrich T, Marsch S, Egli A, et al. Predictors of infectious meningitis or encephalitis: the yield of cerebrospinal fluid in a cross-sectional study. BMC Infect

32. Urban TJ, Shen Y, Stolz A, et al. Limited contribution of common genetic variants to risk for liver injury due to a variety of drugs. Pharmacogenet Genomics

33. Cirulli ET, Nicoletti P, Abramson K, et al. A Missense Variant in PTPN22 is a Risk Factor for Drug-induced Liver Injury. Gastroenterology 2019;156:1707-1716. 
477 34. Liu T, Wang RX, Han J, et al. Comprehensive bile acid profiling in hereditary 478 intrahepatic cholestasis: Genetic and clinical correlations. Liver Int $479 \quad 2018 ; 38: 1676-1685$.

480 35. Chen M, Suzuki A, Borlak J, et al. Drug-induced liver injury: Interactions between 481 drug properties and host factors. J Hepatol 2015;63:503-514.

482 36. Fontana RJ. Pathogenesis of idiosyncratic drug-induced liver injury and clinical 483 perspectives. Gastroenterology 2014;146:914-928.

484 


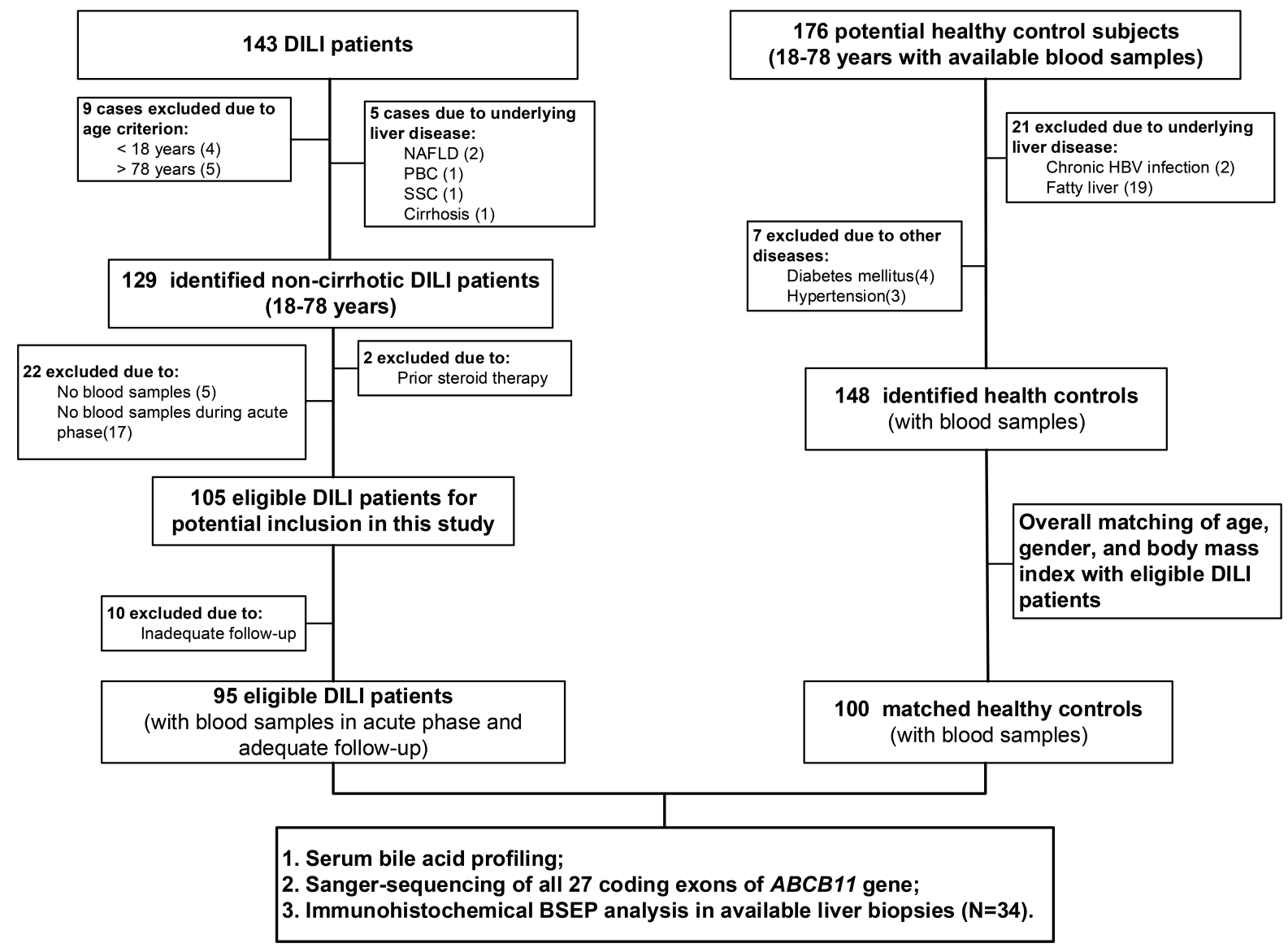



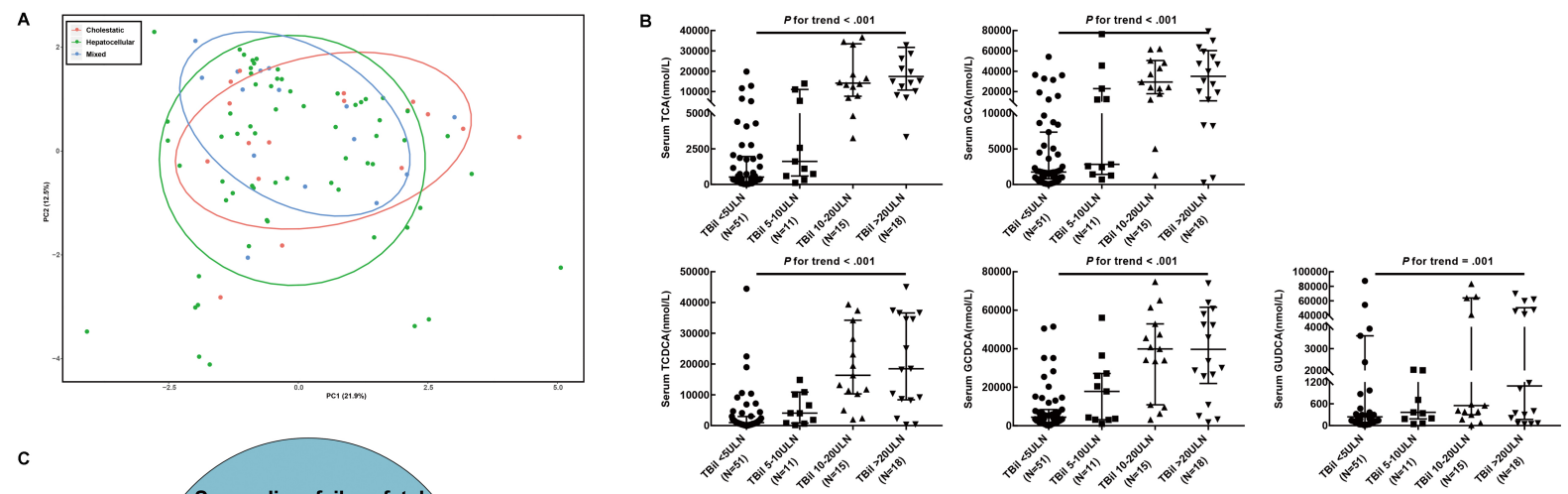

C
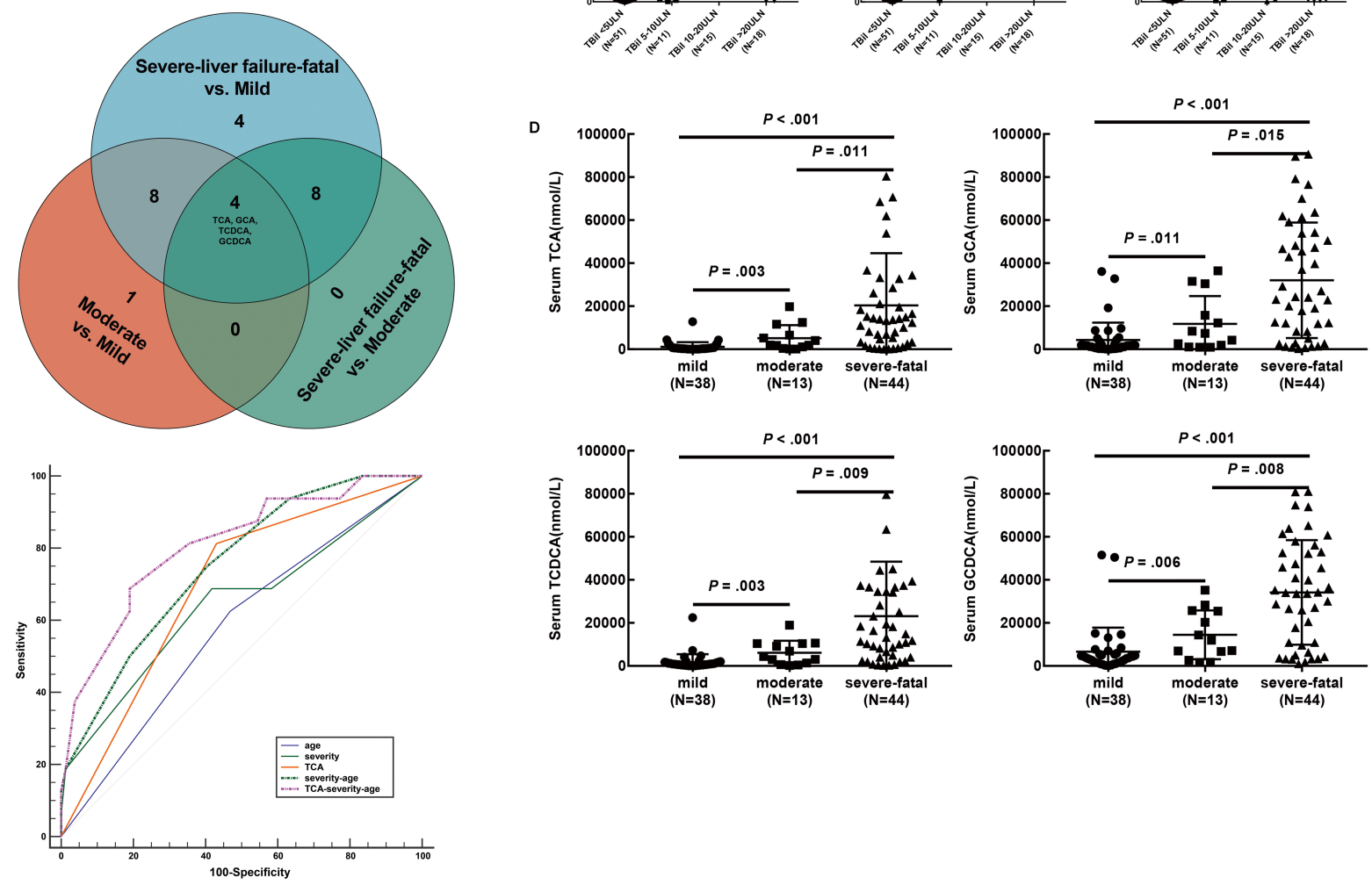

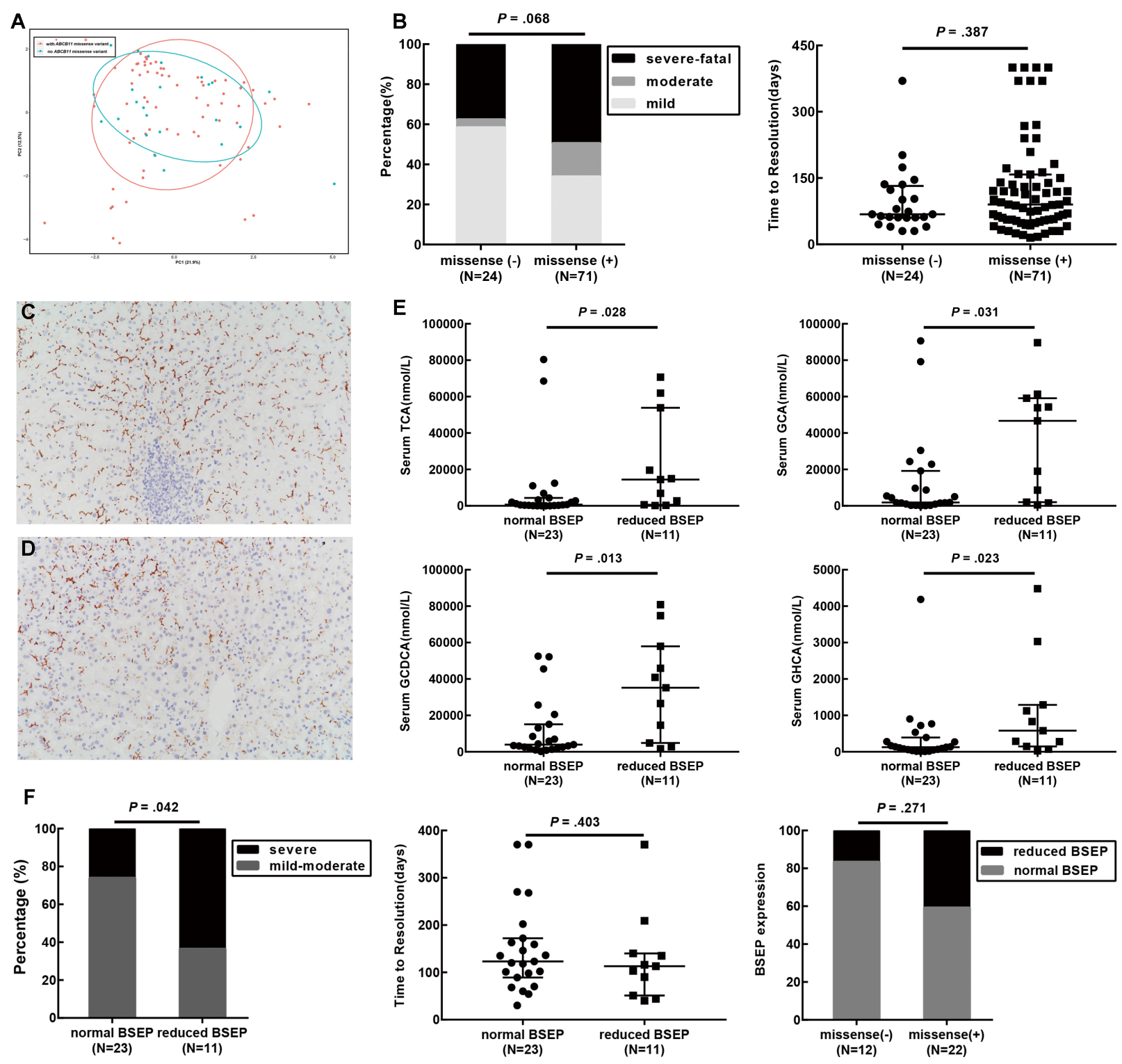
Table 1 Demographics and baseline characteristics of healthy controls and DILI patients with different biochemical phenotypes

\begin{tabular}{|c|c|c|c|c|c|c|}
\hline Indexes & $\begin{array}{l}\text { Healthy } \\
\text { controls } \\
(\mathrm{N}=100)\end{array}$ & $\begin{array}{l}\text { Overall DILI } \\
\qquad(\mathrm{N}=95)\end{array}$ & $\begin{array}{l}\text { Hepatocellular }(\mathrm{R} \geq \mathbf{5}) \\
\qquad(\mathrm{N}=\mathbf{6 3})\end{array}$ & $\begin{array}{c}\text { Mixed }(2<\mathrm{R}<5) \\
\quad(\mathrm{N}=15)\end{array}$ & $\begin{array}{c}\text { Cholestatic }(\mathrm{R} \leq 2) \\
(\mathrm{N}=17)\end{array}$ & $P$ value $\#$ \\
\hline Age (years) & $47(45,55)$ & $53(40,63)$ & $50(38,61)$ & $63(43,66)$ & $51(48,62)$ & .13 \\
\hline Female, $\mathbf{N}(\%)$ & $67(67.0)$ & $70(73.7)$ & 47 (74.6) & $9(60.0)$ & $14(82.4)$ & .34 \\
\hline BMI $\left(\mathrm{kg} / \mathrm{m}^{2}\right)$ & $\begin{array}{c}23.19(21.63, \\
25.50)\end{array}$ & $22.75(20.08,25.00)$ & $22.80(20.15,25.10)$ & $23.20(21.03,25.03)$ & $21.70(19.50,24.00)$ & .45 \\
\hline $\operatorname{ALT}(I U / L)$ & $27(20,33)$ & $492(172,1026)$ & $808(475,1294)$ & $192(111,349)$ & $58(35,79)$ & $<.001$ \\
\hline $\operatorname{AST}(\mathbf{I U} / \mathbf{L})$ & $28(21,33)$ & $311(110,709)$ & $479(264,1020)$ & $132(109,278)$ & $57(43,79)$ & $<.001$ \\
\hline $\operatorname{ALP}(I U / L)$ & $91(67,117)$ & $201(126,262)$ & $175(112,223)$ & $277(124,387)$ & $246(198,406)$ & .004 \\
\hline GGT (IU/L) & $35(29,43)$ & $178(96,298)$ & $169(89,261)$ & $187(87,554)$ & $215(106,307)$ & .46 \\
\hline TBil $(\mu \mathrm{mol} / \mathrm{L})$ & $13(10,16)$ & $60(21,187)$ & $54(21,146)$ & $165(45,245)$ & $61(19,227)$ & .23 \\
\hline DBil $(\mu \mathrm{mol} / \mathrm{L})$ & $8(6,10)$ & $37(7,130)$ & $32(7,93)$ & $113(17,146)$ & $31(6,125)$ & .32 \\
\hline TBA $(\mu \mathrm{mol} / \mathrm{L})$ & $\begin{array}{c}2.57(2.00, \\
4.16)\end{array}$ & $31.85(11.19,151.06)$ & $26.65(9.80,148.68)$ & $29.57(10.63,112.70)$ & $120.81(15.89,248.57)$ & .10 \\
\hline Albumin $(g / L)$ & NT & $38.4 \pm 5.0$ & $38.9 \pm 5.1$ & $37.5 \pm 4.2$ & $37.4 \pm 5.3$ & .56 \\
\hline Globulin $(\mathrm{g} / \mathrm{L})$ & NT & $30.5 \pm 6.8$ & $32.1 \pm 7.3$ & $30.9 \pm 8.3$ & $28.5 \pm 5.7$ & .40 \\
\hline $\operatorname{IgG}(\mathbf{m g} / \mathbf{d l})$ & NT & $1312.9 \pm 343.1$ & $1369.5 \pm 307.8$ & $1309.5 \pm 580.5$ & $1221.9 \pm 379.4$ & .65 \\
\hline IgM (mg/dl) & NT & $98.10(70.68,139.40)$ & $93.55(66.08,140.00)$ & $101.00(75.40,130.00)$ & $108.00(79.30,146.00)$ & .37 \\
\hline INR & NT & $1.11(1.01,1.23)$ & $1.12(1.03,1.23)$ & $1.14(0.89,1.27)$ & $1.04(0.94,1.30)$ & .64 \\
\hline ANA & NT & & & & & NA \\
\hline Negative & & $64(67.4)$ & $46(73.0)$ & $10(66.7)$ & $8(47.1)$ & \\
\hline 1:80 positive & & $20(21.1)$ & $11(17.5)$ & $2(13.3)$ & $7(41.2)$ & \\
\hline 1:160 positive & & $10(10.5)$ & $5(7.9)$ & $3(20.0)$ & $2(11.8)$ & \\
\hline 1:320 positive & & $1(1.1)$ & $1(1.6)$ & $0(0)$ & $0(0)$ & \\
\hline Negative AMA-M2 (N, \%) & NT & $95(100)$ & $63(100)$ & $15(100)$ & $17(100)$ & NA \\
\hline Ceruloplasmin (g/L) & NT & $0.30(0.24,0.35)$ & $0.29(0.24,0.34)$ & $0.29(0.23,0.42)$ & $0.34(0.24,0.53)$ & .27 \\
\hline Ferritin $(\mathbf{n g} / \mathbf{m L})$ & NT & $205.95(128.33,369.00)$ & $212.90(104.90,373.40)$ & $322.20(162.00,530.80)$ & $133.45(98.53,185.10)$ & .022 \\
\hline TS (\%) & NT & $46.93 \pm 19.28$ & $48.65 \pm 21.36$ & $48.35 \pm 14.07$ & $36.56 \pm 10.23$ & .16 \\
\hline $\mathbf{A F P}(\mathbf{n g} / \mathrm{mL})$ & NT & $3.90(2.90,6.24)$ & $4.17(2.98,8.14)$ & $3.56(2.40,4.44)$ & $3.41(2.60,5.13)$ & .109 \\
\hline $\mathrm{WBC}\left(\times 10^{9} / \mathrm{L}\right)$ & $7.1 \pm 1.9$ & $5.6 \pm 2.1$ & $5.7 \pm 2.2$ & $5.7 \pm 1.2$ & $5.5 \pm 2.5$ & .98 \\
\hline Platelets $\left(\times 10^{9} / \mathrm{L}\right)$ & $194.4 \pm 59.6$ & $203.2 \pm 58.3$ & $210.6 \pm 56.5$ & $192.8 \pm 55.9$ & $184.8 \pm 67.7$ & .65 \\
\hline Hemoglobin $(g / L)$ & $128.8 \pm 8.4$ & $132.4 \pm 17.0$ & $135.0 \pm 16.8$ & $133.4 \pm 13.4$ & $123.2 \pm 18.4$ & .34 \\
\hline RUCAM score & NT & $6(5,7)$ & $7(6,8)$ & $5(4,6)$ & $4(4,6)$ & $<.001$ \\
\hline Severity score & NT & $2(1,3)$ & $2(1,3)$ & $3(1,3)$ & $2(1,3)$ & .40 \\
\hline
\end{tabular}


Footnote: ${ }^{P} \boldsymbol{P}$ value stands for the overall comparison of hepatocellular, mixed and cholestatic phenotype of DILI; AFP, alpha fetoprotein;

ALP, alkaline phosphatase; ALT, alanine aminotransferase; AMA-M2, anti-mitochondrial M2 antibody; ANA, anti-nuclear antibody;

AST, aspartate aminotransferase; BMI, body mass index; DBil, conjugated bilirubin; GGT, gamma-glutamyl transferase; Ig,

immunoglobulin; INR, international normalized ratio; NA, not available for statistically significance analysis; NT, not tested; RUCAM,

Roussel Uclaf Causality Assessment Method; TBA, total bile acids; TBil, total bilirubin; TS, transferrin saturation; WBC, white blood

cells. 
Table 2 Serum TCA level was an independent risk factor for days to resolution by Cox-regression analysis

\begin{tabular}{|c|c|c|c|c|}
\hline \multirow{2}{*}{ Significant factors in univariate-analysis } & \multicolumn{2}{|c|}{ Univariate } & \multicolumn{2}{|c|}{ Multivariate } \\
\hline & HR $(95 \%$ CI $)$ & $P$ value & HR $(95 \%$ CI $)$ & $P$ value \\
\hline Severity & $0.75(0.62,0.91)$ & .004 & $0.90(0.69,1.16)$ & .41 \\
\hline TBA $(>31.85 \mu \mathrm{mol} / \mathrm{L}$ vs. $<31.85 \mu \mathrm{mol} / \mathrm{L})$ & $0.65(0.43,0.98)$ & .041 & $0.83(0.41,1.67)$ & .59 \\
\hline GCA $(>5463.00 \mathrm{nmol} / \mathrm{L}$ vs. $<5463.00 \mathrm{nmol} / \mathrm{L})$ & $0.58(0.38,0.88)$ & .010 & $0.49(0.16,1.5)$ & .21 \\
\hline TUDCA (>1073.60nmol/L vs. $<1073.60 \mathrm{nmol} / \mathrm{L})$ & $0.56(0.37,0.85)$ & .007 & $1.06(0.46,2.42)$ & .90 \\
\hline THCA (>109.65nmol/L vs. $<109.65 \mathrm{nmol} / \mathrm{L})$ & $0.59(0.39,0.89)$ & .013 & $0.89(0.41,1.93)$ & .76 \\
\hline TCA (>1955.41nmol/L vs. $<1955.41 \mathrm{nmol} / \mathrm{L})$ & $0.47(0.31,0.73)$ & .001 & $3.04(1.08,8.58)$ & .036 \\
\hline TLCA-3S (>360.40nmol/L vs. $<360.40 \mathrm{nmol} / \mathrm{L})$ & $0.56(0.37,0.87)$ & .009 & $1.11(0.65,1.89)$ & .72 \\
\hline NorCA $(>22.29 \mathrm{nmol} / \mathrm{L}$ vs. $<22.29 \mathrm{nmol} / \mathrm{L})$ & $0.58(0.38,0.87)$ & .009 & $1.40(0.81,2.41)$ & .22 \\
\hline ß CDCA (>23.03nmol/L vs. $<23.03 \mathrm{nmol} / \mathrm{L})$ & $1.82(1.19,2.78)$ & .006 & $0.75(0.39,1.44)$ & .39 \\
\hline
\end{tabular}

Footnote: $\boldsymbol{\beta}$ CDCA, $3 \beta$-chenodeoxycholic acid; CI, Confidence Interval; GCA, glycocholic acid; HR, Hazard Ratio; NorCA, nor cholic acid; TBA, total bile acid; TCA, taurocholic acid; THCA, taurohyocholate; TLCA-3S, taurolithocholic acid 3 sulfate; TUDCA,

tauroursodeoxycholic acid. 
Table 3 Addition of serum TCA level could improve predictive ability of 6-month abnormal biochemistry in DILI patients

\begin{tabular}{|c|c|c|c|c|c|c|c|}
\hline $\begin{array}{l}\text { Evaluatin } \\
\text { g indexes }\end{array}$ & $\begin{array}{l}\text { AUROC } \\
(95 \% \mathrm{CI})\end{array}$ & $\begin{array}{l}\text { Optimal } \\
\text { cut-off } \\
\text { value }\end{array}$ & $\begin{array}{l}\text { Sensitivity } \\
(95 \% \text { CI }), \%\end{array}$ & $\begin{array}{c}\text { Specificity } \\
(95 \% \text { CI }), \%\end{array}$ & $\begin{array}{c}\text { Positive } \\
\text { likelihood } \\
\text { ratio }\end{array}$ & $\begin{array}{c}\text { Negative } \\
\text { likelihood } \\
\text { ratio }\end{array}$ & $\begin{array}{c}P \text { value when } \\
\text { compared with }^{\#}\end{array}$ \\
\hline $\begin{array}{l}\text { Age } \\
\text { (years) }\end{array}$ & $\begin{array}{c}0.58 \\
(0.47-0.68)\end{array}$ & 53 & $\begin{array}{c}62.50(35.40- \\
84.80)\end{array}$ & $\begin{array}{c}53.16(41.60- \\
64.50)\end{array}$ & 1.33 & 0.71 & 0.0044 \\
\hline Severity & $\begin{array}{c}0.65 \\
(0.54-0.74)\end{array}$ & 2 & $\begin{array}{c}68.75(41.30- \\
89.00)\end{array}$ & $\begin{array}{c}58.23(46.60- \\
69.20)\end{array}$ & 1.65 & 0.54 & 0.11 \\
\hline $\begin{array}{l}\text { TCA } \\
(\mathrm{nmol} / \mathrm{L})\end{array}$ & $\begin{array}{c}0.69 \\
(0.59-0.78)\end{array}$ & 1955.41 & $\begin{array}{c}81.25(54.40- \\
96.00)\end{array}$ & $\begin{array}{c}56.96(45.30- \\
68.10)\end{array}$ & 1.89 & 0.33 & 0.0009 \\
\hline $\begin{array}{l}\text { Severity-a } \\
\text { ge }\end{array}$ & $\begin{array}{c}0.75 \\
(0.65-0.83)\end{array}$ & 0.13 & $\begin{array}{c}75.00(47.60- \\
92.70)\end{array}$ & $\begin{array}{c}59.49(47.90- \\
70.40)\end{array}$ & 1.85 & 0.42 & 0.37 \\
\hline $\begin{array}{l}\text { TCA-seve } \\
\text { rity-age }\end{array}$ & $\begin{array}{c}0.81 \\
(0.71-0.88)\end{array}$ & 0.17 & $\begin{array}{c}68.75(41.30- \\
89.00)\end{array}$ & $\begin{array}{c}81.01(70.60- \\
89.00)\end{array}$ & 3.62 & 0.39 & I \\
\hline
\end{tabular}

Footnote: AUROC, areas under the receiver operating characteristic curves; CI, confidence interval; TCA, taurocholic acid. 